\title{
$\wedge$ Madridge
}

madridge Journal of Internal and Emergency Medicine

Interconnecting Scientific World

Review

Open Access

\section{Deadly Communicable Diseases and "Superbugs" Brought to Western Societies Could Exacerbate Cardiovascular Disease Worldwide and why a newly Discovered Biologic may Ameliorate These Challenges: A Potential Important wake-up call}

\author{
Burton M Altura ${ }^{1-6 *}$ \\ ${ }^{\prime}$ Department of Physiology and Pharmacology, State University of New York, USA \\ ${ }^{2}$ Department of Medicine, University of New York, USA \\ ${ }^{3}$ The Center for Muscle and Cardiovascular Disease, USA \\ ${ }^{4}$ The School of Graduate Studies in Molecular and Cellular Science. The State University of New York, Downstate Medical Center, \\ Brooklyn, New York, USA \\ ${ }^{5}$ Bio-Defense Systems, Inc, Rockville Centre, New York, USA \\ ${ }^{6}$ Orient Biomedical, Estero, Florida, USA
}

\section{Article Info}

*Corresponding author:
Burton M Altura
Professor
Department of Physiology and
Pharmacology
SUNY Downstate Medical Center
Brooklyn, New York
USA
Tel: 718-270-2194
E-mail: burton.altura@downstate.edu

Received: May 14, 2018

Accepted: May 21, 2018

Published: May 28, 2018

Citation: Altura BM. Deadly Communicable Diseases and "Superbugs" Brought To Western Societies Could Exacerbate Cardiovascular Disease Worldwide and why A Newly Discovered Biologic may Ameliorate these Challenges: A Potential Important wake-up call. Madridge J Intern Emerg Med. 2018; 2(2): 56-60.

doi: 10.18689/mjiem-1000112

Copyright: (c) 2018 The Author(s). This work is licensed under a Creative Commons Attribution 4.0 International License, which permits unrestricted use, distribution, and reproduction in any medium, provided the original work is properly cited.

Published by Madridge Publishers
Keywords: Cardiovascular diseases; Superbugs; Infectious Diseases; Syphilis, Gonorrhea, Leprosy.

\section{Introduction}

The major threat to health worldwide is atherosclerosis [1,2]. Cardiovascular diseases (CVDs), emanating from atherosclerosis, such as myocardial infarction, strokes, peripheral vascular diseases, untreatable, resistant hypertension, and premature deaths are an enormous burden on healthcare costs worldwide; the financial burdens are becoming impossible to pay for. Although there are numerous risk factors for development of atherogenesis and CVDs [for reviews, see 1,2], infectious microorganisms are usually not taken into consideration as causal agents in development of atherogenesis and CVDs [1,2]. However, numerous studies, often overlooked, have become links to atherogenesis, e.g., Bacillus typhosus (first suggested more than 100 years ago) $[3,4]$, Chlamydia pneumoniae [5-8], Helicobacter pylori [9], syphilis [10], Cytomegalovirus [10], herpes simplex [11], and other viruses [12,13]. Over the past decade, there have been suggestions that diverse antibiotics may prove useful in the prevention and treatment of atherogenesis and CVDs [14,15]. In view of the potential, important contributions of infectious microorganisms to etiology of atherosclerosis and CVDs, we believe it may be critical to consider the potential illegal immigration to the USA, UK, and Europe.

There has been a growing concern over the increasing numbers of illegal immigrants to Western societies and the concomitant widespread increase in dangerous, communicable diseases [16-18]. In the United States, alone, more than 1,000,000 illegal immigrants/ year have entered the country over the past few years. More than threefourths of these people come from Mexico, El Salvador, Guatemala, and Honduras.

Legal immigrants and refugees are required to undergo a medical examination for migration to the United States, while they still are residing in their home countries 
overseas. These examinations are carried out under the auspices of "The Centers for Disease Control and Prevention $(C D C)$ ", which provide explicit details/instructions to the Panel Physicians who conduct the examinations.

These procedures consist of a physical examination, an evaluation (skin test/chest $x$-ray exam) for tuberculosis (TB), and blood test for syphilis. Requirements for vaccinations are based on recommendations from the "Advisory Committee on Immunization Practices". Individuals who do not pass the examination(s) due to certain health conditions are denied admission to the United States. Such conditions, for failure, include drug addiction or any type(s) of communicable diseases of public health concern, e.g., TB, syphilis, gonorrhea, leprosy, and ever-changing threats such as polio, cholera, diphtheria, smallpox or severe respiratory syndromes. Unfortunately, illegal immigrants crossing into the United States could, and often do, bring many of these dangerous threats to Western societies. Such an unchecked situation not only has brought dangerous outbreaks of diseases into the United States, and elsewhere, but has often overloaded and stretched healthcare facilities beyond their abilities to take care of its legal citizens and legal refugees. This situation has exposed legal citizens and legal refugees to diseases that have been almost totally eradicated, some being highly contagious, as with TB, drug-resistant TB and polio.

As of this year, the flu has become widespread in the entire continental United States, in one of the worst seasons of the illness in almost a decade according to the CDC. So far, almost 150 U.S. children have died from the flu during this endemic. Even though many individuals have taken the flu vaccine, it is said, unlike in previous years, to be only $25-30 \%$ -effective. However, is this due to the egg-based system mostly used to make the vaccine in the United States or to the $\mathrm{H} 3 \mathrm{~N} 2$ flu strain, or to what we believe is the overloading of the legal citizens and refugees with millions of undocumented aliens, who have crossed illegally over the porous Southern border that have brought deadlier/mutated strains of the flu? Australia was hit-hard during this past winter by the H3N2 flu strain, where their vaccine was said to be only $10 \%$-effective [19]. We believe these increased infections, seen in both the U.S. and Australia, might explain, in part, the major cause of the increased infectious viral strain (s) brought into these countries by illegal migrants.

\section{The Return of Deadly Diseases Wiped-out Years Ago}

Nine diseases that were almost totally eradicated in the U.S. have been making a comeback, i.e., TB, measles, whooping cough, mumps, scarlet fever, dengue fever, leprosy, malaria and bubonic plague $[16-18,20]$. TB is a very infectious disease that spreads through the air. When infected people cough, sneeze, talk or spit, they expel TB germs (i.e., bacilli) into the air. Any person infected with TB spreads through these avenues enough bacilli to infect TB, on average, to about 1015 people/year. Mexico and Central American countries, as of today, have at least 10-times the rate of TB prevalence as does the U.S. Many African countries along with Afghanistan, Cambodia, The Philippines, and Indonesia exhibit rates of TB that are more than 100-150-times that of the U.S. Undocumented, illegal individuals who are disease carriers are costing the U.S. and Western countries billions of dollars per year in healthcare costs. Approximately $70 \%$ of all TB cases, in the USA, are currently found in foreign -born individuals. It has been estimated that treatment of only one case of TB costs over $\$ 250,000 /$ year and sometimes as much as $\$ 1,200,000 /$ year in the U.S. [6]. And even with such high costs, for treatment, about half of these infected TB patients in the U.S. have multi-drug-resistant TB and will die prematurely [16-18,21,22]. Minnesota has experienced an unusual rise of measles cases which they have not seen in 20 years [23]. Somali refugees in Minnesota have been identified as responsible for almost $25 \%$ of the active cases of TB in the state [8]. According to the CDC, in 2014 there were 599 cases of measles reported in 22 states, whereas in 2012 there were only 55 cases [20]. The current case count for whooping cough in the USA has increased to almost 35,000 while in 1976 the number was 1,010 [20]. Maricopa county on the border of Arizona, which received more than 200 of the immigrant children in the summer of 2014 recorded recordbreaking cases of infectious diseases, including almost 600 cases of MRSA, over 400 cases of Strep, more than 200 cases of whooping cough, and almost 200 cases of viral aseptic meningitis [9]. Illinois in 2014 experienced almost 150 cases of mumps while in 2013, the number was 25 [5,9]. In Maine, Governor Lepage remarked that "the biggest problem in our state", is the asylum seekers who are responsible for a large increase in TB, HIV, hepatitis $C$, and hepatitis $B$ cases $[10,11]$. In 2016, the CDC reported 16 cases of bubonic plague in the US, four of whom died. In 2005, only four cases were reported with no deaths [20]. The obvious question for legalized citizens, refugees and healthcare personnel, is what can we do to protect people against the contraction of one or more of these infectious diseases, particularly as it is almost impossible to vaccinate people against all of these potential bacilli, viruses, and other infectious organisms? Another important question, are these disease carriers causing unprecedented rises in atherogenesis (which often takes some years to develop) which will, and are, producing increased incidences in certain CVDs.

\section{Early Development and Use of Flu Vaccines}

Approximately 85 years ago, the first flu vaccine was made by Jonas Salk and Thomas Francis after it was discovered that viruses (influenza virus types $A, B$, and $C$ rarely) cause flu [see 12, for review]. It was first utilized to protect the U.S. military forces against the flu in World War II [12]. The most dangerous (virulent) influenza, the 1918 Spanish flu, pandemic infected about $5 \%$ of the world's population and killed approximately $2 \%$ of the world's population. In an attempt to prevent a pandemic, and an increased risk of Guillain- Barre syndrome (i.e., approximately one to nine cases per million doses), $25 \%$ of the people, in 1979, in the U.S. A. were given the vaccine [13]. Since that time, influenza vaccines have been vastly improved in design, at least until the past year. Highly pathogenic avian influenza viruses of the $\mathrm{H} 5$ and $\mathrm{H} 3$ subtypes 
are a current, serious problem for poultry and human health. Despite the advent of drugs like oseltamivir, and other antiflu therapies, severe influenza still kills tens of thousands in the U.S.A. every year and millions worldwide. How many of these deaths are due to infection from illegal migrants is not known.

\section{Complications Caused By "Superbugs"}

A disturbing trend in antimicrobial -antiviral resistance is the advent of "Superbugs" which often complicates the treatment of flu-immunocompromised and TBimmunocompromised patients. Since many of these patients have underlying CVDs, this results in patients who are very difficult to treat. To this must be added the numerous hospitalizations and increased morbidity from contaminated meats, vegetables, seafoods, and dairy products. Many of the emerging types of avian flus (e.g., H1N1, H2N2, H3N2, A $\{\mathrm{H} 10 \mathrm{~N} 8]$ ) along with $T B$, dengue fever, and bubonic plague have a hemorrhagic component to them which complicates effective treatment. Any new effective treatments against these infections should be able to prevent/ameliorate these types of hemorrhages, particularly in the lungs. Currently, government resources are being overstretched and often remain powerless to combat flus, drug-resistant TB and the other diseases mentioned herein, particularly with the increased numbers of illegal migrants coming to Western societies.

\section{Many Infectious Diseases are characterized by Hemorrhagic Components and "Cytokine Storms" in The Lungs}

Many of the infectious diseases, discussed herein, demonstrate patients presenting with high fevers, coughs, headaches, muscle and joint pain, and severe fatigue [14]. However, when the lungs become infected and severely inflamed, by an overproduction of a host of mediators (primarily by regulatory epithelial cells and alveolar macrophages), i.e., cytokines or chemokines (e.g., interferons, tumor necrosis factor, interleukins, macrophage factors, etc.), this gives rise to what is termed a "cytokine storm" [15]. These "cytokine storms" often proceed unabated, to cause severe tissue damage and hemorrhages, followed by death which is preceded by multiple organ failure, triggered by a spillover of the cytokines and chemokines into the general circulation, particularly in the lungs, kidneys, and cardiovascular system [14-17]. These inflammatory responses are triggered as the infected cells die via apoptosis and necrosis. These deadly scenarios have attracted numerous investigators, worldwide, to devise therapies to either prevent or stem the courses of events leading to the "cytokine storms" which result in massive release of multiple cytokines and chemokines [1820].

\section{Discovery and Development of a New Biologic Host- defense Factor, HDFx}

Our laboratories, for more than 30 years, have been working on a new approach to develop host-defense factors that stimulate various arms of the innate and adaptive immune systems. To this end, we have discovered, a new host-defense factor we have termed "HDFx", that is a conserved protein found, so far, in rats, mice, rabbits, guineapigs, dogs, and subhuman primates [21-25]. Since HDFx is a conserved protein, we believe it must be present in humans.

Approximately 135 years ago, Elie Metchnikoff, the father of immunology, hypothesized that the body, under adverse conditions, would produce powerful immunostimulants which could act on different arms of the immune system and serve to protect the host against major insults, inflammatory conditions, and diseases [25]. Metchnikoff's early studies pointed to the important contributions of macrophages and phagocytic leukocytes to natural (innate) resistance against pathogenic bacteria and viruses. Over the past 40 years, considerable evidence has accumulated to support a strong relationship between the functional (physiological) state of the microcirculation, macrophages-phagocytes, alveolar macrophages, natural killer (NK) cells, the reticuloendothelial system, platelets, and "pit cells" in the liver to host defense and resistance to pathogens, trauma, sepsis, wounding, circulatory shock and combined injuries [26-34]. Ongoing studies from our laboratories have shown that HDFX is protective (to different degrees) against a variety of insults ranging from hemorrhage, trauma, endotoxins, a variety of bacteria (e.g., E. coli. S. enteriditis, C. welchii, among others), combined injuries, and centripetal forces to septic shock [2124]. A unique attribute of HDFx is its ability to accelerate wound healing [24]. Most importantly, HDFx has been demonstrated, in several animal models, to inhibit release of multiple cytokines and chemokines, including TNF-alpha, IL6, IL-8, IL-1beta, IFN-gamma, and numerous macrophage factors [36,39-44]. Thus, HDFX can either prevent or ameliorate "cytokine storms" induced by both gram-negative and grampositive bacteria, toxic fungal microorganisms, trauma, systemic inflammatory conditions, tissue damage, blood loss, and sepsis, among other dangerous bodily insults [36,39-44]. We have also produced some preliminary data to suggest that HDFx might be useful in the treatment and amelioration of hemorrhagic fevers [45].

\section{HDFx and Its Potential against "Superbugs"}

Gram-negative "Superbugs" appear to be major culprits in hospital-borne infections [2,17, and 29]. These "superbug" -induced infections seem to be more difficult to kill than gram-positive bacteria because they are protected by "double cell membranes". So, in order to kill the gram-negative bacteria (e.g., induced by $\mathrm{TB}$, diptheria, bubonic plague, syphilis, E. coli, S. enteriditis, whooping cough, etc.) most of the approaches have been to design antibiotics to penetrate these membrane barriers. In our opinion, another likely approach would be to engulf the bacteria, viruses, and fungi and let "supercharged" macrophages, phagocytic leukocytes, NK cells, "pit cells", and platelets digest and destroy these microorganisms. But, in order for this to occur, in an expeditious manner, it is our belief that the microcirculation to key tissue-organs (i.e., lungs, kidneys, spleen, bone marrow, 
heart) must perforce produce optimal capillary blood flows and distribution. Thus, an ideal drug or therapeutic molecule would be to stimulate several arms of innate immune system. So, to our knowledge, only HDFx appears to be the only molecule that embodies all these attributes and demonstrate therapeutic qualities against several classes of "Superbugs". Most importantly, HDFx can also accelerate wound healing in internal organs that could be damaged (e.g., hemorrhges) by dangerous, infectious microorganisms [38].

\section{Conclusions and Future Thoughts}

It is rather obvious from the facts presented, herein, that the Western world is under a clear assault from the migration of undocumented -illegal people, who are bringing many contagious diseases that were eliminated in Western Societies until the past several years. This migration of millions of diseased individuals to the USA and elsewhere has overburdened the healthcare systems, and infected thousands of people who have already died due to these undocumented disease carriers. Often overlooked by the medical community is the growing importance and threats of infectious microorganisms to development of atherosclerosis and CVDs, This situation clearly represents a "present and clear danger". If new drugs/vaccines are not designed to combat many of these drug-resistant illnesses, and administered to legal citizens and refugees as well as to undocumented people, this could result in numerous worldwide plagues and exacerbation of CVD processes with the spread (and development of atherogenesis leading to more CVDs), thus, potentially resulting in the killing of millions of people, and susceptible children, as the healthcare systems would not be able to handle such huge numbers of infected individuals. In addition, it must be noted that the monetary costs would be prohibitive.

Having such a disastrous situation in mind, our laboratories have been working on a new approach to the prevention and treatment of multiple diseases and "Superbugs" carried by diseased individuals. More than 30 years of research, done by our laboratories, has resulted in the discovery of a new, indigenous biologic host-defense molecule which we have termed HDFx. HDFx is a conserved protein found in every mammalian species so far investigated. It possesses many unique qualities including the ability to accelerate wound healing. With proper funding, it is our hope to have its complete molecular structure elucidated in the near future and ready for testing in human subjects.

\section{Acknowledgements}

Some of our research efforts described, herein, were supported, in part, by Research Grants from the National Institutes of Health and unrestricted grants from several pharmaceutical companies. Our studies could not have been done without the collaboration of many individuals: A. Gebrewold, A. Carella, C.Thaw, R.W. Burton, C. Parillo, and B.T. Altura. Several ideas and experiments needed for our discovery and development of "HDFx" were carried out while the author was on the Faculties of New York University School of Medicine and The Albert Einstein College of Medicine of Yeshiva University.

\section{References}

1. Lilly LS. Pathophysiology of Heart Disease. Wolters Kluwer, Philadelphia. 2016.

2. Kumar V, Abbas AK, Aster JC. Robbins and Cotran Pathologic Basis of Disease. Elsevier Saunders, Philadelphia 2015.

3. Gilbert A, Lion G. Arterites infectieuses experimentales. Comptes Rendus Hebdomadaires des Seances et Memoires de la Societe de Biologie. 1889; 41: 583-584.

4. Nieto FJ. Infections and atherosclerosis: new clues from an old hypothesis? Am J Epidemiol. 1998; 48: 937-948. doi: 10.1093/oxfordjournals.aje. a009570

5. Beatty WL, Morrison RP, Byrne GI. Persistent chlamydiae: from cell culture to a paradigm for chlamydial pathogenesis. Microbiol Rev. 1994; 58: 686-699.

6. Dunne M. The evolving relationship between Chlamydia pneumoniae and atherosclerosis. Current Opin Infect Dis. 2000; 13(6): 583-591.

7. Gupta S, Leatham EW, Carrington D, Mendall MA, Kaski JC, et al. Circulation. 1997; 96: 404-407.

8. Hu H, Pierce GN, Zhing G. The atherogenic effects of Chlamydia are dependent on serum cholesterol and specific to Chlamydia pneumonia. J Clin Invest. 1999; 103: 747-753. doi: 10.1172/JCI4582

9. Danesh J. Coronary heart disease, Heliobacter pylori, dental disease, Chlamydia pneumoniae, and cytomegalovirus: meta-analyses of prospective studies. Am Hear J. 1999; 138: 434-437. doi: 10.1016/S0002-8703(99)70270-X

10. Fuster V, Kelly DD. Promoting Cardiovascular Health in the Developing World. The National Academies Press. Washington, DC. 2010.

11. Dunne M. Infectious Agents and Cardiovascular Disease. National Academies Press. 2004.

12. Garfinkel EG, Bozovich G, Duroca A, Beck E, Mautner B. Randomised trial of roxithromycin in non-Q-wave coronary syndromes: ROXIS Pilot Study Group. Lancet. 1997; 350: 404-407. doi: 10.1016/S0140-6736(97)07201-2

13. Nieto FJ. Viruses and atherosclerosis: a critical review of the epidemiologic evidence. Am Heart J. 1999; 138: 453-460. doi: 10.1016/S00028703(99)70274-7

14. Anderson JL, Muhlestein JB, Carlquist J, Allen A, Trehan S, et al. Randomized secondary prevention trial of azithromycin in patients with coronary artery disease and serological evidence for Chlamydia pneumonia infection: the axithromycin in coronary artery disease: elimination of myocardial infection with Chlamydia (ACADEMIC) study. Circulation. 1999; 99: 1540-1547.

15. Fong IG, Chiu B, Vira E, Jang D, Fong MW, et al. Can an antibiotic (macrolide) prevent Chlamydia pneumoniae - induced atherosclerosis in a rabbit model? Clin Diagnostic Lab Immunol. 1999; 6: 891-894.

16. U.S. Army War College. The Dark Side of Illegal Immigration: Cause for National Concern. 2014.

17. Cohen J, Powderly WG. Opal SM Infectious Diseases. Elsevier. 2016.

18. Carter S. 'Potential for a Public Health disaster': Illegal immigrant surge leaves officials with 'no idea' which diseases are coming across. The Blaze. 2014.

19. McKay B. Flu virus extends its reach. Wall Street Journal. 2018; 13-14, p A3.

20. Adams DA, Thomas KR, Jajosky JR, Foster L, Barol G, et al. Summary of Notifiable Infectious Diseases-United States-2015. Morbity and Mortality Weekly Report-United States 2015. 2017; 64(53): 1-143.

21. Marks SM, Flood J, Seaworth B, Hirsch-Moverman Y, Armstrong L, et al. Treatment practices, outcomes, and costs of multidrug-resistant and extensively drug-resistant tuberculosis, United States, 2005-2007. Emerging Infect Dis J. 2014; 20(5).

22. Millen MC. Discovering Tuberculosis. Yale University Press, New Haven. 2015. 
23. Howard J. Minnesota measles outbreak exceeds last year's nationwide numbers. CNN. 2017.

24. Jackson T. Special report: CDC knew resurgence in diseases from refugees and immigrants was dangerous and deadly. News Legit. 2017.

25. LePage, Governor P Town Hall Meeting, Augusta. 2016.

26. Disease Control and Prevention Data Infectious Diseases. The Maine Center for Disease Control and Prevention, Augusta. 2016.

27. Salk JE, Menke WJ, Francis T. A clinical, epidemiological and immunological evaluation of vaccination against epidemic influenza. Am J Epidemiol. 1945; 42: 57-93. doi: 10.1093/oxfordjournals.aje.a119025

28. Taubenberger JK, Morens DM. 1918 Influenza: the mother of all pandemics. Emerg Infect Dis. 2006; 12: 15-22. doi: 10.3201/eid1201.050979

29. Murphy CK, weaver C. Janeway's Immunology. Garland Science. 2016.

30. Liu Q, Zhou YH, Yang ZQ. The cytokine storm of severe influenza and development of immunomodulatory therapy. Cell Molec Therap. 2016; 11: 3-10. doi: 10.1038/cmi.2015.74

31. D'Ella RV, Harrison K, et al. Targeting the: cytokine stotm" for therapeutic benefit. Clin Vaccine Immunol. 2013; 20: 319-327. doi: 10.1128/CVI.00636-12

32. Knipe DM, Howley PM. Fields Virology, Walters Kluwer/Lippincott Williams \& Wilkins Health, Philadelphia. 2013.

33. Liu Q, Liu DY, Yang ZQ. Characteristics of human infection with avian influenza viruses and development of new antiviral agents. Acta Pharmacol. 2016; 3(4): 1257-1269. doi: 10.1038/aps.2013.121

34. Iwasaki A, Medzhitov R. A new shield for cytokine storm. Cell. 2011; 46: 861-862. doi: 10.1016/j.cell.2011.08.027

35. Ramos I, Fernandez -Sesma A. Modulating the innate response to influenza a virus: potential therapeutic use of anti-inflammatory drugs. Fron Immunol. 2015; 6: 361. doi: 10.3389/fimmu.2015.00361

36. Altura BM, Gebrewold A, Carella A. A novel biologic immunomodulator, HDFx, protects against lethal hemorrhage, endotoxins and traumatic injury: potential relevance to emerging diseases. Int J Clin Exp Med. 2009; 2: $266-279$.
37. Altura BM, Carella A, Gebrewold A. HDFx: a novel biologic immunomodulator is therapeutically-effective in hemorrhagic and intestinal-ischemic shock: importance of microcirculatory -immunological interactions and their potential implications for the warfighter and disaster victims. Int J Clin Exp Med. 2011; 4: 331-340.

38. Altura BM, Carella A, Gebrewold A. HDFx: a novel biologic immunomodulator accelerates wound healing and is suggestive of unique regenerative powers for the warfighter and disaster victims. Int J Clin Exp Med. 2012; 5: 289-295.

39. Altura BM, Gebrewold A, Carella A. A recently discovered biologic and its potential use in prevention and treatment of hemorrhagic fever viruses and antibiotic-resistant "Superbugs". J Haematol Thromboembolic Dis. 2016; 4: 100252.

40. Altura BM, Gebrewokd A, CarellaA,Altura BT.HDFx:A novel immunomodulator and potential fighter against cytokine storms in inflammatory and septic conditions in dogs and farm animals. Int J Vet Health Sci Res. 2016; 5: 1-3.

41. Altura BM, Altura BT. HDFx: A novel biologic immunomodulator for potential control and treatment of NK cell and macrophage dysfunction in drug-resistant tuberculosis. J Clin Med Ther. 2017; 2: 20.

42. Altura BM, Altura BT. Why a recently-discovered host-defense factor, HDFx, may ameliorate and prevent inflammatory lesions induced by sarcoidosis. Madridge J immunol. 2018; 2(1): 40-42.

43. Altura BM, Altura BT. HDFx for the prevention and treatment of vasodilatory septic shock: A personal perspective. Vasc Dis and Ther. 2017; 2(6): 1-3.

44. Altura BM, Altura BT. Use of HDFx, a novel immunomodulator, to stop the germs from winning in hospitals and on the battlefields: The dangers of antibiotic resistance. Int J Vaccines and Res. 2017; 4(1): 1-2.

45. Altura BM, Gebrewold A, Carella A. HDFx: A recently discovered biologic and its potential use in prevention and treatment of hemorrhagic fever viruses and antibiotic-resistant "Superbugs". J hematol Thromboembol Dis. 2016; 4: 100252. 